\title{
Analysis of the $(Q, r)$ Inventory Model for Perishables with Positive Lead Times and Lost Sales
}

\author{
Emre Berk \\ Faculty of Business Administration, Bilkent University, Bilkent 06800, Ankara, Turkey, \\ eberk@bilkent.edu.tr \\ Ülkü Gürler \\ Faculty of Engineering, Department of Industrial Engineering, Bilkent University, Bilkent 06800, Ankara, Turkey, \\ ulku@bilkent.edu.tr
}

\begin{abstract}
We consider a perishable inventory system with Poisson demands, fixed shelf lives, constant lead times, and lost sales in the presence of nonnegligible fixed ordering costs. The inventory control policy employed is the continuous-review $(Q, r)$ policy, where $r<Q$. The system is modeled using an embedded Markov process approach by introducing the concept of the effective shelf life of a batch in use. Using the stationary distribution of the effective shelf life, we obtain the expressions for the operating characteristics and construct the expected cost rate function for the inventory system. Our numerical study indicates that the determination of the policy parameters exactly as modeled herein results in significant improvements in cost rates with respect to a previously proposed heuristic. We also compare the $(Q, r)$ policy with respect to a time-based benchmark policy and find that the $(Q, r)$ policy might be impractical for rare events, but overall appears to be a good heuristic policy.
\end{abstract}

Subject classifications: inventory: perishables; lot size-reorder point policy; lost sales; effective shelf life. Area of review: Manufacturing, Service, and Supply Chain Operations.

History: Received May 2003; revisions received September 2004, February 2006, September 2006; accepted

December 2006.

\section{Introduction}

Groceries, pharmaceuticals, composite materials, sheet metal, and blood and its derivatives are a few examples of perishable goods found in a wide variety of industries. Due to their common occurrence and importance, a broad literature has developed over the years on management of perishable inventories. However, the existing literature is insufficient in addressing the mostly encountered setting where items have fixed shelf lives, replenishment times are constant, and ordering costs are not negligible.

In this paper, we provide the analysis of a continuousreview perishable inventory system with constant shelf lives, fixed ordering costs, and constant lead times under the $(Q, r)$ policy with the restriction $r<Q$, which implies at most one outstanding order at any time. With Poisson demands and lost sales, we model the system as an embedded Markov process and introduce the concept of effective shelf life, which corresponds to the remaining shelf life of items on hand at the instances when the inventory level hits $Q$. Based on the stationary distribution of the effective shelf life, we derive the operating characteristics of the system and construct the expected cost rate function. We provide comparisons of the $(Q, r)$ policy with a threeparameter time-based control policy and the only existing heuristic (Chiu 1995) available for the lost-sales $(Q, r)$ model. Our numerical results indicate that the performance of the lot size-reorder policy is good overall, but deteriorates for high service levels, and that determination of the policy parameters exactly as proposed herein results in significant cost savings. We believe that the effective shelf life concept introduced in our work may also enable analysis of other models and help in developing new control policies and heuristics.

The structure of the optimal policy for perishables with fixed lifetimes in the presence of nonnegligible lead times remains an open question. In view of the previous work on periodic-review systems (e.g., Fries 1975, Nahmias 1975), it may be conjectured that the optimal continuous-review control policy should make use of the information regarding the current inventory levels, the remaining shelf lives of the items in stock, and the remaining lead times of the outstanding orders. Even if it were found, it is unlikely that anyone interested in a real problem would be able to use such a complex optimal policy (Schmidt and Nahmias 1985). The researchers have therefore focused on lot sizereorder policies as a reasonable alternative policy class, although not necessarily optimal.

There are a few works in the literature on perishables under continuous review with fixed lifetimes. With zero lead times, Weiss (1980) shows that the $(s, S)$ policy class is optimal for Poisson demands. For recent studies with negligible lead times, see Liu and Lian (1999), Lian and Liu (2001), and Gürler and Özkaya (2003). With constant 
lead times, Schmidt and Nahmias (1985) provide the first exact analytical treatment of a system with fixed shelf lives. They consider a lost-sales system with zero ordering costs under the $(S-1, S)$ control policy. Perry and Posner (1998) extend the $(S-1, S)$ model to the case of lead-time dependent partial backordering. When ordering costs are nonnegligible, there is no exact treatment in the literature for fixed lifetimes and constant lead times. Chiu (1995) and Lian and Liu (2001) provide only approximations to the $(Q, r)$ model.

The rest of this paper is organized as follows. Section 2 introduces the basic assumptions of our model. Section 3 defines and establishes certain properties of the effective shelf-life process, and derives its stationary distribution. The expressions for the operating characteristics of the inventory system and the expected cost rate function are developed in $\$ 4$. In $\S 5$, we present our numerical study and, finally, we conclude in $\$ 6$ with a brief summary of our work.

\section{Basic Assumptions}

We consider the following inventory system. Unit external demands are generated according to a Poisson process with rate $\lambda$. Replenishment is done in batches, and there is a fixed, positive procurement lead time, $L$. All of the items in a batch have identical lifetimes. After joining stock, a batch has a constant shelf life of $\tau$ time units, beyond which it is no longer usable. The items are withdrawn from stock to satisfy the demand according to the FIFO policy. Each unit held in stock incurs a holding cost $h$ per unit time, and each unit that perishes incurs a cost of $p$. All unmet demand is lost at a unit lost-sales cost of $\pi$. There is a fixed nonzero ordering cost $K$. The operational objective under the inventory control policy is to minimize the total expected costs per unit time. We employ the following continuous-review lot size-reorder point inventory control policy.

Policy: A replenishment order of size $Q$ is placed whenever the inventory position hits $r$ by demand, or drops to zero by perishing, whichever occurs first.

Note that when a finite lifetime is introduced, it is possible that the items in a batch on hand perish before the inventory position hits exactly $r$ at a demand occurrence. Hence, we modify the reordering decision slightly and allow for a reorder to be placed at the perishing instances. We assume that $0 \leqslant r<Q$. This restriction implies that there will be at most one outstanding order at any given point in time. Hence, we can use inventory levels interchangeably with inventory positions. We first proceed with the assumption $\tau>L$. A couple of observations will be helpful in our analysis. First, there may be at most two different batches on hand at any time; but due to the FIFO rule, the older batch will be consumed first, either through demand or by perishing, while the younger batch ages on the shelf. Second, there is always an instance when the inventory level hits $Q$ after the outstanding order is received. If a stockout has been experienced during the lead-time period, then the inventory level is immediately raised to $Q$ as the outstanding order joins stock. At this instant, there is only one batch of items in stock with the remaining lifetime of all the items being exactly $\tau$. If, however, a stockout is not experienced during the lead time, the inventory is raised to a level between $Q+1$ and $Q+r$ upon the arrival of the outstanding order. In this case, the younger batch will age on the shelf until the older batch is depleted first. As the last item in the older batch is sold or the batch perishes, the inventory level drops to $Q$. At this instance, the younger batch is the only batch in stock and has a remaining lifetime strictly smaller than $\tau$. Therefore, if one observes the remaining lifetimes of the on-hand items at the instances when the inventory level hits $Q$, they would appear as a sequence of random variables, which will be referred to as the sequence of effective shelf lives. Next, we obtain the probability distribution and certain properties for this sequence.

\section{Effective Shelf-Life Distribution}

In this section, we show that the effective shelf-life sequence has the Markov property and establish certain properties leading to the ergodicity of the process, and derive the stationary effective shelf-life distribution.

Suppose we start to observe the inventory system operating under the proposed $(Q, r)$ policy at time $t=0$, with $Q$ fresh items on hand. Let $\left\{T_{n}, n \geqslant 1\right\}$ be the sequence of time epochs at which the inventory level hits $Q$ for the $n$th time, with $T_{1}=0$. Then, $I\left(T_{n}\right)=Q$ for all $n \geqslant 1$, where $I(t)$ is the inventory level at time $t$. Furthermore, let $\left\{Z_{n}, n \geqslant 1\right\}$ be the sequence of effective shelf lives of the items at $T_{n}$ where, without loss of generality, $Z_{1}=\tau$. Considering the system between two consecutive instances at which the inventory level hits $Q$, we develop the expressions for the limiting probability distribution of the effective shelf-life sequence $\left\{Z_{n}, n \geqslant 1\right\}$.

We first demonstrate that the effective shelf-life process $\left\{Z_{n}, n \geqslant 1\right\}$ possesses the Markov property, which is a crucial assumption for the validity of our modeling approach.

Let $\left\{Y_{j}, j \geqslant 1\right\}$ be the sequence of Poisson demand arrival times in chronological order and let $N(t)$ be the counting process of the arrivals in $(0, t]$. Then, $\left\{T_{n}, n \geqslant 1\right\}$ is a sequence of stopping times for $N(t)$. Hence, the time between the $N\left(T_{n}\right)+j$ th demand arrival and the last stopping time, i.e., $Y_{N\left(T_{n}\right)+j}-T_{n}$, has an Erlang $j$ distribution with rate $\lambda$, independent of the events prior to $T_{n}$. We refer to the time between $T_{n+1}$ and $T_{n}$ as the $n$th embedded cycle for $n \geqslant 1$. Note that the inventory system discussed above is a regenerative one, and the regeneration points are those instances where the inventory level is raised to $Q$ after a stockout and the remaining shelf lives of these items are $\tau$. However, an analysis based on the regenerative cycles defined via these regeneration epochs is quite 
Figure 1. Possible cycle realizations for the $(Q, r)$ model.

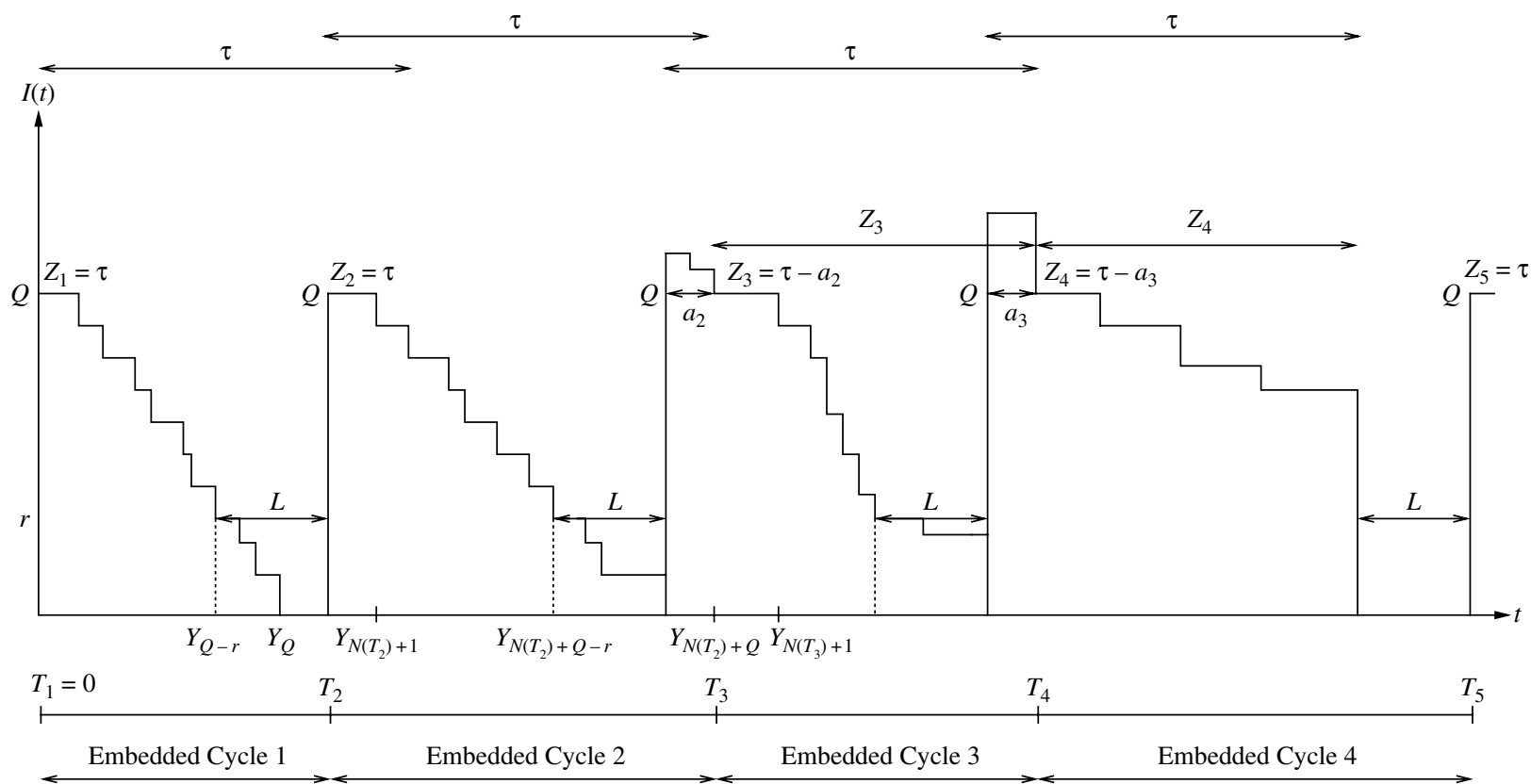

intractable. We therefore base our approach on the embedded process instead. Referring to Figure 1, we illustrate a possible realization of the system dynamics.

We set the beginning of Embedded Cycle 1 at $T_{1}$ as the time origin, $t=0$. In Embedded Cycle 1, the inventory level drops to $r$ after $Q-r$ demands have arrived and a replenishment order is given at $t=Y_{Q-r}$. During the lead-time period of length $L$, the remaining $r$ units are also depleted by demand and Embedded Cycle 1 is completed at the end of the lead-time period, starting Embedded Cycle 2 with a fresh batch of $Q$ items $\left(T_{2}=Y_{Q-r}+L\right)$. This realization is characterized by the events $Y_{Q}<Z_{1}=\tau$ and $Y_{Q}-Y_{Q-r}<L$, resulting in $Z_{2}=\tau$. In the second embedded cycle, the inventory level drops to $r$ at time $t=Y_{N\left(T_{2}\right)+Q-r}$ and an order of size $Q$ is placed. At the end of the lead time, there are still some unsold items left over. Those items are then depleted by demand at time $t=Y_{N\left(T_{2}\right)+Q}$ without perishing, and Embedded Cycle 3 starts $\left(T_{3}=Y_{N\left(T_{2}\right)+Q}\right)$. This realization is characterized by the events $Y_{N\left(T_{2}\right)+Q-r}-T_{2}<Z_{2}, Y_{N\left(T_{2}\right)+Q}-Y_{N\left(T_{2}\right)+Q-r}>L$, and $Y_{N\left(T_{2}\right)+Q}-T_{2}<Z_{2}$. Letting $a_{n}$ denote the time between the end of the lead time and the start of the $(n+1)$ st embedded cycle, we see from Figure 1 that $a_{2}=\left(Y_{N\left(T_{2}\right)+Q}-T_{2}\right)-$ $\left(Y_{N\left(T_{2}\right)+Q-r}-T_{2}\right)-L=Y_{N\left(T_{2}\right)+Q}-Y_{N\left(T_{2}\right)+Q-r}-L$ and $Z_{3}=\tau-a_{2}=\tau-\left[Y_{N\left(T_{2}\right)+Q}-Y_{N\left(T_{2}\right)+Q-r}\right]+L$. Hence, at the beginning of Embedded Cycle 3, the remaining shelf life of the $Q$ items on hand is $Z_{3}$. In this embedded cycle, the inventory level drops to $r$ at $t=Y_{N\left(T_{3}\right)+Q-r}$ and an order of size $Q$ is placed. At the end of the lead time, there are still unsold items. Unlike the previous case, however, some of these leftover items perish at time $t=T_{4}$ before they are depleted by demand. This perishing event completes the third embedded cycle and starts the fourth one $\left(T_{4}=T_{3}+Z_{3}\right)$. This realization is characterized by the events $Y_{N\left(T_{3}\right)+Q}-T_{3}>Z_{3}$, $Y_{N\left(T_{3}\right)+Q-r}-T_{3}<Z_{3}$, and $Y_{N\left(T_{3}\right)+Q}-Y_{N\left(T_{3}\right)+Q-r}>L$, and results in $a_{3}=Z_{3}-\left(Y_{N\left(T_{3}\right)+Q-r}-T_{3}\right)-L$ and $Z_{4}=\tau-$ $a_{3}=\tau-\left[Z_{3}-\left(Y_{N\left(T_{3}\right)+Q-r}-T_{3}\right)-L\right]$. The process continues in this fashion.

As illustrated by the foregoing discussion, the effective shelf life $Z_{n+1}$ at the beginning of the $(n+1)$ st embedded cycle is completely determined by (i) $Z_{n}$ and, (ii) the Poisson demand arrival process after the stopping time $T_{n}$. Therefore, the embedded process $\left\{Z_{n}, n \geqslant 1\right\}$ has the Markov property. Furthermore, for $n \geqslant 2, Y_{N\left(T_{n}\right)+Q-r}-T_{n}$ and $Y_{N\left(T_{n}\right)+Q}-Y_{N\left(T_{n}\right)+Q-r}$ are independent Erlang $(Q-r)$ and Erlang $r$ variables with rate $\lambda$, respectively. Defining $X_{r}=Y_{N\left(T_{n}\right)+Q}-Y_{N\left(T_{n}\right)+Q-r}, X_{Q}=Y_{N\left(T_{n}\right)+Q}-T_{n}$, and $X_{Q-r}=Y_{N\left(T_{n}\right)+Q-r}-T_{n}$ for brevity, we have

$Z_{n+1}=\left\{\begin{array}{cc}\tau-X_{r}+L & \text { if } X_{r}>L, X_{Q}<Z_{n}, \\ \tau-\left(Z_{n}-X_{Q-r}-L\right) & \quad \text { if } Z_{n}<X_{Q}, X_{r}>L, X_{Q-r}<Z_{n}-L, \\ \tau \quad & \text { otherwise. }\end{array}\right.$

From (1) follows an interesting observation that is not intuitively obvious. The support of the effective shelf-life distribution in any cycle is the interval $(L, \tau]$, that is, $Z_{n+1}>L$ for all $Z_{n}, n \geqslant 1$.

Next, we consider the transition probability function of the process $Z_{n}$. Let $\left\{F_{n}(z), n \geqslant 1\right\}$ denote the sequence of distribution functions of the effective shelf lives, which are mixture distribution functions, continuous on the interval $(L, \tau)$, with a positive mass, $P_{n}(\tau)$, at $\tau$. (Henceforth, the integral of any function $U(\cdot)$ w.r.t. $F_{n}$ should 
be understood as $\int_{0}^{\tau} U(x) d F_{n}(x)=\int_{0}^{\tau-} U(x) F_{n}(x) d x+$ $U(\tau) P_{n}(\tau)$.) Also, for any d.f. $F$, we have $\bar{F}=1-F$. For $j \geqslant 1$, let $H_{j}$ and $h_{j}$ denote the distribution and the density functions of an Erlang type $j$ variable with rate $\lambda$. Let $\mathscr{X}=(L, \tau]$ be the state space of the remaining shelflife sequence, and for fixed $x \in \mathscr{X}$ consider the stochastic transition function $p(A \mid x)$ as a probability measure for $A \in \mathscr{F}_{\mathscr{X}}$, where $\mathscr{F}_{\mathscr{X}}$ is the Borel $\sigma$-field generated by the subsets of $\mathscr{X}$. Without loss of generality, consider the Borel sets of type $A=(L, z], z \in(L, \tau)$ (note that if $z=\tau$, the probability of set $A$ is one). Then, we have:

THEOREM 1. (a) For $L<z<\tau$ and $x \leqslant \tau$,

$$
\begin{aligned}
p(A \mid x) & =P\left(Z_{n+1} \leqslant z \mid Z_{n}=x\right) \\
& =\bar{H}_{r}(\tau+L-z) H_{Q-r}(z-L-(\tau-x)), \\
P\left(Z_{n+1}\right. & \left.=\tau \mid Z_{n}=x\right)=1-\bar{H}_{r}(L) H_{Q-r}(x-L) .
\end{aligned}
$$

(b) For $L<z<\tau$,

$F_{n+1}(z)$

$=\bar{H}_{r}(\tau+L-z) \int_{\tau+L-z}^{\tau} H_{Q-r}(z-L-\tau+x) d F_{n}(x)$

$=\bar{H}_{r}(\tau+L-z) \int_{0}^{z-L} \bar{F}_{n}(\tau+L-z+x) d H_{Q-r}(x)$,

and for $z=\tau, P_{n+1}(z)=1-F_{Z_{n+1}}(\tau-)$.

Proof. Referring to (1), we can write the transition function as

$$
\begin{aligned}
P(A \mid x)= & P\left(Z_{n+1} \in A ; X_{r}>L, X_{Q}<x\right) \\
& +P\left(Z_{n+1} \in A ; x<X_{Q} ; X_{r}>L ; X_{Q-r}<x-L\right) \\
= & P\left(X_{Q-r}<z-L-\tau+x ; X_{r}>\tau+L-z\right) \\
= & \bar{H}_{r}(\tau+L-z) H_{Q-r}(z-L-\tau+x),
\end{aligned}
$$

from which (2) follows. The complement of the probability in (2) as $z \rightarrow \tau^{-}$gives (3). Part (b) is obtained from part (a) by unconditioning.

Next, we establish certain properties of the effective shelf-life process leading to its ergodicity.

Proposition 1. (i) For a given demand sequence $X_{1}$, $X_{2}, \ldots, X_{Q}$, in the embedded cycle $n, Z_{n+1}$ is a nonincreasing function of $Z_{n}$.

(ii) $\mathrm{E}\left[Z_{n+1} \mid Z_{n}=x\right]$

$$
=\tau-\int_{0}^{x-L} \bar{H}_{r}(x-u) H_{Q-r}(u) d u .
$$

(iii) Let $0<\delta<\tau-L$ be chosen such that $\epsilon=$ $\int_{0}^{\tau-L-\delta} \bar{H}_{r}(\tau-y) H_{Q-r}(y) d y-\delta>0$. Then,

$\mathrm{E}\left[Z_{n+1} \mid Z_{n}=x\right]<x-\epsilon$ for $x \in(\tau-\delta, \tau]$.
Proof. See the online appendix, which is available as part of the online version that can be found at http://or.pubs. informs.org/.

Proposition 2. Let $\mu_{\tau}$ denote the expected time between two consecutive instances at which the inventory level hits $Q$ with effective shelf life $\tau$ (i.e., two consecutive fresh starts). Then,

$\mu_{\tau} \leqslant \frac{(\tau+L)}{\left[1-\bar{H}_{r}(L) H_{Q-r}(\tau-L)\right]}$.

Proof. See the online appendix.

The above proposition implies positive recurrence. It also gives the expected number of stockouts per unit time, $1 / \mu_{\tau}$, for a given $(Q, r)$ pair. Finally, we have the ergodicity result.

THEOREM 2. The process $\left\{Z_{n}, n \geqslant 1\right\}$ is ergodic.

Proof. We first show that the process $\left\{Z_{n}, n \geqslant 1\right\}$ is irreducible. From Theorem 1 , we have $P\left(Z_{n+1}=\tau \mid Z_{n}=x\right)$ $>0$ and $p(A \mid x=\tau)=\bar{H}_{r}(\tau+L-z) H_{Q-r}(z-L)>0$. Hence, starting from $x$, the process enters to any set $A$ in two transitions with positive probability. We now follow Laslett et al. (1978), referred to as LPT henceforth. Let $\phi$ be the Lebesgue measure over the interval $(L, \tau)$. From the above argument, the process is $\phi$-irreducible. Also, the conditional probability distribution of $Z_{n+1}$ given $Z_{n}=x$ is continuous over the interval $(\tau+L-x, \tau)$ with a probability mass at $z=\tau$. Let $f_{n}(z \mid x)$ be the corresponding p.d.f. over the continuous part and $p_{n}(\tau \mid x)$ be the point mass at $\tau$. Then, after LPT, for any continuous, bounded function $g$,

$$
\begin{aligned}
P g(x) \equiv & \int g(z) d F_{n}(z \mid x) \\
= & \int_{\tau+L-x}^{\tau} g(z) f_{n}(z \mid x) d x+g(\tau) p_{n}(\tau \mid x) \\
= & \int_{\tau+L-x}^{\tau} g(z) d\left[\bar{H}_{r}(\tau+L-z) H_{Q-r}(z-L-\tau+x)\right] \\
& +g(\tau)\left[1-\bar{H}_{r}(L) H_{Q-r}(x-L)\right],
\end{aligned}
$$

which is continuous and bounded. Hence, the sequence $\left(Z_{n}, n \geqslant 1\right)$ is weakly continuous and by Theorem 4.1 of LPT, any bounded set $B$ with positive Lebesgue measure is a test set. We also have $\mathrm{E}\left[Z_{n+1} \mid Z_{n}=x\right] \leqslant \tau$, and by Proposition 1(iii), there exists $\epsilon>0$ such that $\mathrm{E}\left[Z_{n+1} \mid Z_{n}=x\right] \leqslant x-\epsilon$ for $x \in B^{c}=(\tau-\delta, \tau]$. Hence, by Theorem 2.2 of LTP, the mean hitting times are bounded, which implies the ergodicity of the process.

Theorem 2 ensures that the limiting distribution for the remaining shelf-life process exists. Letting $F$ denote this limiting distribution function, we have the following result in conjunction with Theorem 1(b). 
Corollary 1. For $L<z<\tau$,

$$
\begin{aligned}
F(z) & =\int_{\tau+L-z}^{\tau} \bar{H}_{r}(\tau+L-z) H_{Q-r}(z-L-\tau+x) d F(x) \\
& =\int_{0}^{z-L} \bar{H}_{r}(\tau+L-z) \bar{F}(\tau+L-z+x) d H_{Q-r}(x) .
\end{aligned}
$$

So far, we have assumed that $\tau>L$. Now, suppose that $\tau \leqslant L$. This implies that the process will always start with a fresh batch at the end of the lead time. Then, $F_{n}(z)=$ $F(z)=0$ if $z<\tau$ and 1 if $z \geqslant \tau$. Hence, this case is practically uninteresting and analytically simple. Nevertheless, the results of the next section also apply to this case with $F_{n}$ given above.

\section{Operating Characteristics and the Objective Function}

In this section, we obtain the expressions for the operating characteristics of the inventory system at hand and construct the objective function of the decision model.

Operating Characteristics. We derive the expressions for the operating characteristics of the inventory system for a given value $Z=z$ of the effective shelf life at steady state.

We begin with the length, $C L$, of an embedded cycle at steady state:

$$
C L= \begin{cases}X_{Q-r}+L & \text { if } X_{Q}<X_{Q-r}+L, X_{Q}<z, \\ X_{Q-r}+L & \text { if } z-L<X_{Q-r}<z, X_{Q}>z, \\ X_{Q} & \text { if } X_{Q-r}+L<X_{Q}<z, \\ z & \text { if } X_{Q-r}<z-L, X_{Q}>z, \\ z+L & \text { if } X_{Q-r}>z .\end{cases}
$$

The first two events above correspond to fresh batch arrivals when the inventory is depleted during the lead time. The next one corresponds to the event that all $Q$ units are depleted after the lead time and before they perish. The one before the last indicates that the batch perishes after the lead time and the last one indicates that an order is placed due to perishing of the items before the reorder point $r$ is reached. Carrying out the expectations, we have

$\mathrm{E}[C L \mid Z=z]$

$$
\begin{aligned}
= & L+\eta(Q, r)+z \bar{H}_{Q-r}(z) \\
& +\frac{Q-r}{\lambda}\left[H_{Q-r+1}(z)-H_{Q-r+1}(z-L)\right]+\gamma(Q, r),
\end{aligned}
$$

where

$$
\begin{aligned}
& \gamma(Q, r)=H_{Q-r}(z-L)\left[z-L \bar{H}_{r}(L)-\frac{r}{\lambda} H_{r+1}(L)\right], \\
& \eta(Q, r)=\int_{0}^{z-L}\left[\frac{r}{\lambda} H_{r+1}(z-x)-(z-x) H_{r}(z-x)\right] d H_{Q-r}(x) .
\end{aligned}
$$

Note that $\eta(Q, r)$ corresponds to the expected time that a received batch stays in stock until the previous one perishes or is depleted.

Let the total stock time in a cycle (i.e., the area under the inventory-level curve within an embedded cycle) be denoted by $O H$ and let $\{N(t), t \geqslant 0\}$ be the number of demand arrivals in an interval of length $t$, where the time origin is taken to be the cycle beginning. We have

$O H= \begin{cases}\sum_{i=1}^{Q} X_{i} & \text { if } X_{Q}<X_{Q-r}+L, X_{Q}<z, \\ \sum_{i=1}^{N(z)} X_{i}+z[Q-N(z)] & \text { if } z-L<X_{Q-r}<z, X_{Q}>z, \\ \sum_{i=1}^{Q} X_{i}+Q\left[X_{Q}-X_{Q-r}-L\right] & \text { if } X_{Q-r}+L<X_{Q}<z, \\ & \text { if } X_{Q-r}<z-L, X_{Q}>z, \\ \sum_{i=1}^{N(z)} X_{i}+z[Q-N(z)]+Q\left[z-X_{Q-r}-L\right] & \text { if } X_{Q-r}>z .\end{cases}$

Taking the expectations, we have

$$
\begin{aligned}
& \mathrm{E}[O H \mid Z=z] \\
& =Q\left[\eta(Q, r)+z \bar{H}_{Q}(z)-\frac{Q-r}{\lambda} H_{Q-r+1}(z-L)\right. \\
& \left.\quad+\frac{(Q+1)}{2 \lambda} H_{Q+1}(z)+\gamma(Q, r)\right]-\frac{\lambda z^{2}}{2} \bar{H}_{Q-1}(z) .
\end{aligned}
$$

The number of lost sales in an embedded cycle, $L S$, is as follows:

$L S= \begin{cases}N\left(X_{Q-r}+L-X_{Q}\right) & \text { if } X_{Q}<X_{Q-r}+L, X_{Q}<z, \\ N\left(X_{Q-r}+L-z\right) & \text { if } z-L<X_{Q-r}<z, X_{Q}>z, \\ N(L) & \text { if } X_{Q-r}>z .\end{cases}$

The first event corresponds to the inventory being depleted by demand during the lead time; the second corresponds to the inventory on hand perishing during the lead time, and the last event is that the on-hand items perish before the reorder level is reached. Then,

$$
\begin{aligned}
& \mathrm{E}[L S \mid Z=z] \\
& =\lambda\left[L+\eta(Q, r)-z\left(H_{Q-r}(z)-H_{Q}(z)\right)+\gamma(Q, r)\right] \\
& \quad+(Q-r)\left[H_{Q-r+1}(z)-H_{Q-r+1}(z-L)\right]-Q H_{Q+1}(z) .
\end{aligned}
$$


Finally, we have the number of perishing items in an embedded cycle, $P$, such that $P=Q-N(z)$ if $X_{Q}>z$ and zero otherwise. Then,

$\mathrm{E}[P \mid Z=z]=Q \bar{H}_{Q}(z)-\lambda z \bar{H}_{Q-1}(z)$.

The above expressions are for a given value of $Z=z$. Unconditioning on $z$, we can obtain the operating characteristics of the inventory system within an embedded cycle at steady state.

Objective Function. The objective for our problem is the minimization of the expected cost rate, $T C$. The construction of the expected cost rate builds on two previously established results. First, we invoke a result of Ross (1970), that two average cost criteria as defined below are equivalent. Then, following Tijms (1994), we show that for the inventory system at hand, the expected average cost based on regenerative cycles can be written based on embedded cycles.

We begin with the equivalence result of Ross for a general semi-Markov decision process (SMDP). In our model, the remaining shelf-life process $\left\{Z_{n}, n \geqslant 1\right\}$ has the state space given by $\mathscr{X}=(L, \tau]$, and the action space consists of a single stationary action that places an order of size $Q$ when the inventory position crosses $r$. The process $\left\{Z_{n}, n \geqslant 1\right\}$ regenerates itself whenever $Z_{n}=\tau$. Without loss of generality, we assume that $Z_{1}=\tau$ at $t=0$. Let $C(t)$ denote the cost incurred over the interval $(0, t]$, and $C_{i}\left(Z_{i}, \mathbf{X}\right)$ and $L_{i}\left(Z_{i}, \mathbf{X}\right)$ be the cost and the length of the $i$ th $(i=1,2, \ldots)$ embedded cycle, where $\mathbf{X}$ denotes the array of interarrival times of Poisson demands within the $i$ th embedded cycle, independent of $\left(Z_{1}, \ldots, Z_{i}\right)$. Following Ross, we define the two average cost criteria and a necessary condition as follows:

$$
\begin{aligned}
\phi_{1}(\tau)= & \varlimsup_{t \rightarrow \infty} \mathrm{E}\left[C(t) / t \mid Z_{1}=\tau\right], \\
\phi_{2}(\tau)= & \varlimsup_{n \rightarrow \infty} \mathrm{E}\left[\sum_{i=1}^{n} C_{i}\left(Z_{i}, \mathbf{X}\right) \mid Z_{1}=\tau\right] / \\
& \mathrm{E}\left[\sum_{i=1}^{n} L_{i}\left(Z_{i}, \mathbf{X}\right) \mid Z_{1}=\tau\right] .
\end{aligned}
$$

Condition 1. For every state $z \in \mathscr{X}$, there is a positive probability of at least $\epsilon>0$ that the transition time (length of an embedded cycle in our model) will be greater than some $\delta>0$.

The condition above ensures that the transitions of $Z_{n}$ do not take place too quickly. Next, we invoke the following theorem.

Theorem 3 (Ross 1970, Theorem 1). Suppose that Condition 1 holds and the expected length of a regenerative cycle is finite. Then,

$$
\begin{aligned}
\phi_{1}(\tau) & =\phi_{2}(\tau) \\
& =\frac{\mathrm{E}\left[\text { cost of a regenerative cycle } \mid Z_{1}=\tau\right]}{\mathrm{E}\left[\text { length of a regenerative cycle } \mid Z_{1}=\tau\right]} .
\end{aligned}
$$

Referring to Equation (9), we immediately see that, for any starting remaining shelf life $z \in(L, \tau]$, the length $C L$ of an embedded cycle satisfies $L<C L \leqslant \tau+L$. Hence, if we let $\delta=L$, Condition 1 holds for any $0<\epsilon<1$. The requirement on the finiteness of the expected length of a regenerative cycle is also satisfied in our case because by Proposition 2 , the mean time $\mu_{\tau}$ between two fresh starts (i.e., $z=\tau$ ) is bounded from above. Therefore, we have the equivalence of $\phi_{1}(\tau)$ and $\phi_{2}(\tau)$ for our problem.

Next, we show that the expected cost rate based on regenerative cycles can be written based on embedded cycles in conjunction with the limiting effective shelf-life distribution. Let $\mathscr{C}(z)=\mathrm{E}\left[C_{i}\left(Z_{i}, \mathbf{X}\right) \mid Z_{i}=z\right]$ and $\mathscr{L}(z)=\mathrm{E}\left[L_{i}\left(Z_{i}, \mathbf{X}\right) \mid\right.$ $Z_{i}=z$ ] for $i \geqslant 1$. The expectations are independent of the index $i$ when $Z_{i}=z$ is given and are calculated with respect to the interarrival times of Poisson demands $\mathbf{X}$, as provided in Equations (10), (12), (14), and (15).

THEOREM 4. Let $F$ be the limiting distribution function of $\left\{Z_{i}, i \geqslant 1\right\}$. Then,

$\phi_{2}(\tau)=\frac{\int_{L}^{\tau} \mathscr{C}(z) d F(z)}{\int_{L}^{\tau} \mathscr{L}(z) d F(z)}$.

Proof. Follows from the generalization of Tijms (1994) to continuous state spaces and is provided in the online appendix.

From Theorems 3 and 4, we now construct the expected cost rate, $T C$, as follows:

$T C=\frac{K+\int_{z=L}^{\tau}(\theta \mathrm{E}[O H \mid Z=z]+p \mathrm{E}[P \mid Z=z]+\pi \mathrm{E}[L S \mid Z=z]) d F(z)}{\int_{z=L}^{\tau} \mathrm{E}[C L \mid Z=z] d F(z)}$.

\section{Numerical Results and Discussion}

In our numerical study, we examined (i) the sensitivity of the optimal policy parameters to the environmental parameters, (ii) the performance of the proposed policy to that of a more complex one, and (iii) the benefit of computing the $(Q, r)$ policy parameters as developed herein as opposed to using an available heuristic.

Our findings on sensitivity are intuitive and consistent with expectations. The optimal reorder point is nondecreasing in $\pi$ and $\tau$. The optimal order size is increasing in $\tau$ and $\pi$. Longer shelf lives impact the sensitivity of $Q^{*}$ to $\pi$ more than mean interdemand times. As $K$ increases, the optimal order size increases, asymptotically approaching an insensitivity threshold because the perishing costs dominate from then on.

In the absence of a true optimal policy class, the performance of the $(Q, r)$ policy can only be compared against other more sophisticated policies that utilize more information in making reordering decisions. As a reasonable alternative to the optimal policy, we employed a continuous-review three-parameter control policy that considers not only the inventory position, but also the remaining shelf life of the batch currently in use. This benchmark policy $(Q, r, T)_{\mathrm{bm}}$ is as follows: place an order of size $Q$ 
whenever the inventory position crosses $r$ or the remaining shelf life of the batch currently in use hits $T$, whichever occurs first. The policy uses two order trigger mechanisms: one inventory based and the other time based. As such, it contains the $(Q, r)$ policy as a special case when $T$ is set equal to $\tau$.

To assess the utility of the determination of the $(Q, r)$ policy parameters as proposed herein, we also made a comparison with a heuristic suggested in Chiu (1995). The Chiu heuristic has been developed as a by-product of the full backordering case, and is the only available heuristic for determining the $(Q, r)$ policy parameters for a lost-sales perishable inventory system as considered herein (Chiu 1995, Equations (6)-(9), pp. 97-98). It has not been tested before; hence, our numerical study provides a test of the Chiu heuristic for the lost-sales environment for the first time.

For our comparisons, we used a test bed based on Chiu (1995): 24 problems therein and eight additional ones. For consistency with Chiu (1995), we modify, in (20), the ordering cost as $K=\hat{K}+c \cdot Q$, where $\hat{K}$ and $c$ are the fixed and variable components of ordering cost associated with each order of size $Q$.

The steady-state distribution of the effective shelf life in our model was obtained by evaluating integro-differential Equation (8) numerically via discretization over the domain of $Z$. Hence, the computational complexity of our model is governed by that of solving a system of linear equations. For optimization, we used exhaustive search over a broad range of policy parameter values, and observed unimodality in all of the cases considered. The best parameters of the benchmark policy $\left(Q^{*}, r^{*}, T^{*}\right)_{\mathrm{bm}}$ and the corresponding cost rate $T C_{\mathrm{bm}}^{*}$ were obtained via a simulation study conducted with five runs of 10,000 demand arrivals, in which $r$ is allowed to exceed $Q$. The optimal parameters of the lot size-reorder policy $\left(Q^{*}, r^{*}\right)_{e}$ were determined exactly as modeled herein, and the approximate policy parameters $\left(Q^{*}, r^{*}\right)_{a}$ were computed using the Chiu heuristic. We use $\% \Delta_{e}$ and $\% \Delta_{a}$ to denote the percentage deviations of the $(Q, r)$ policy as computed exactly and approximately from the benchmark, $T C_{\mathrm{bm}}^{*}$, respectively.

Tables 1 and 2 tabulate the results for the test problems. The performance of the Chiu heuristic worsens as $\hat{K}$ gets large. The performance of the $\left(Q^{*}, r^{*}\right)_{e}$ policy with respect to the benchmark policy over all cases considered is very good: the average deviation is $0.60 \%$ with a maximum of $3.52 \%$. (See Table 2 for the overall summary.)

From a managerial perspective, it is of interest to identify the operating regions where the $(Q, r)$ policy would perform acceptably (or, conversely, undesirably badly) with

Table 1. Comparison of the benchmark, exact and approximate $(Q, r)$ policies for $\lambda=10, L=1, \tau=3$, and $h=1$.

\begin{tabular}{|c|c|c|c|c|c|c|c|c|c|c|}
\hline Problem no. & $\pi$ & $p$ & $\hat{K}$ & $c$ & $\left(Q^{*}, r^{*}, T^{*}\right)_{b \mathrm{~m}}$ & $T C_{\mathrm{bm}}^{*}$ & $\left(Q^{*}, r^{*}\right)_{e}$ & $\% \Delta_{e}$ & $\left(Q^{*}, r^{*}\right)_{a}$ & $\% \Delta_{a}$ \\
\hline 1 & 20 & 5 & 10 & 5 & $(14,13,2.64)$ & 71.03 & $(15,14)$ & 0.12 & $(14,14)$ & 0.29 \\
\hline 2 & 20 & 5 & 50 & 5 & $(24,12,1.08)$ & 91.86 & $(21,12)$ & 0.64 & $(22,11)$ & 0.82 \\
\hline 3 & 20 & 5 & 100 & 5 & $(26,10,1.08)$ & 112.23 & $(24,11)$ & 1.26 & $(22,11)$ & 2.34 \\
\hline 4 & 40 & 5 & 10 & 5 & $(13,15,0.24)$ & 72.74 & $(15,15)$ & 0.17 & $(14,15)$ & 0.26 \\
\hline 5 & 40 & 5 & 50 & 5 & $(22,13,1.2)$ & 94.89 & $(20,15)$ & 0.83 & $(20,14)$ & 1.01 \\
\hline 6 & 40 & 5 & 100 & 5 & $(25,13,1.08)$ & 116.82 & $(23,13)$ & 1.72 & $(24,12)$ & 2.24 \\
\hline 7 & 20 & 5 & 10 & 15 & $(15,11,1.68)$ & 169.06 & $(15,11)$ & 0.02 & $(15,10)$ & 0.11 \\
\hline 8 & 20 & 5 & 50 & 15 & $(22,8,0.36)$ & 188.35 & $(22,8)$ & 0.01 & $(29,0)$ & 5.09 \\
\hline 9 & 20 & 5 & 100 & 15 & $(24,0,0.12)$ & 206.16 & $(24,0)$ & 0.08 & $(31,0)$ & 4.71 \\
\hline 10 & 40 & 5 & 10 & 15 & $(13,15,0.24)$ & 172.72 & $(12,15)$ & 0.07 & $(13,15)$ & 0.07 \\
\hline 11 & 40 & 5 & 50 & 15 & $(20,13,1.2)$ & 196.05 & $(19,13)$ & 0.09 & $(19,13)$ & 0.09 \\
\hline 12 & 40 & 5 & 100 & 15 & $(24,12,1.08)$ & 218.34 & $(23,12)$ & 0.72 & $(23,11)$ & 1.02 \\
\hline 13 & 20 & 15 & 10 & 5 & $(14,13,2.64)$ & 71.24 & $(14,13)$ & 0.23 & $(13,14)$ & 0.56 \\
\hline 14 & 20 & 15 & 50 & 5 & $(21,12,0.96)$ & 93.54 & $(21,11)$ & 0.39 & $(21,11)$ & 0.39 \\
\hline 15 & 20 & 15 & 100 & 5 & $(24,10,0.96)$ & 115.09 & $(23,10)$ & 0.44 & $(26,8)$ & 4.05 \\
\hline 16 & 40 & 15 & 10 & 5 & $(13,15,0.24)$ & 73.04 & $(13,15)$ & 0.25 & $(13,15)$ & 0.25 \\
\hline 17 & 40 & 15 & 50 & 5 & $(19,14,1.08)$ & 97.19 & $(19,14)$ & 0.15 & $(19,13)$ & 0.40 \\
\hline 18 & 40 & 15 & 100 & 5 & $(24,12,1.08)$ & 120.06 & $(22,13)$ & 1.43 & $(22,12)$ & 2.33 \\
\hline 19 & 20 & 15 & 10 & 15 & $(15,11,1.68)$ & 169.14 & $(15,11)$ & 0.02 & $(15,10)$ & 0.09 \\
\hline 20 & 20 & 15 & 50 & 15 & $(21,7,1.08)$ & 188.89 & $(21,7)$ & 0.02 & $(28,0)$ & 5.74 \\
\hline 21 & 20 & 15 & 100 & 15 & $(22,0,0.12)$ & 206.95 & $(22,0)$ & 0.03 & $(30,0)$ & 6.01 \\
\hline 22 & 40 & 15 & 10 & 15 & $(12,15,0.36)$ & 172.95 & $(12,15)$ & 0.04 & $(12,15)$ & 0.04 \\
\hline 23 & 40 & 15 & 50 & 15 & $(19,13,1.44)$ & 197.25 & $(19,13)$ & 0.04 & $(18,13)$ & 0.22 \\
\hline 24 & 40 & 15 & 100 & 15 & $(23,11,1.08)$ & 220.98 & $(22,11)$ & 0.54 & $(22,11)$ & 0.54 \\
\hline \multicolumn{8}{|c|}{ Average } & 0.39 & & 1.61 \\
\hline \multicolumn{8}{|c|}{ Standard deviation } & 0.49 & & 1.99 \\
\hline \multicolumn{8}{|c|}{ Median } & 0.16 & & 0.55 \\
\hline \multicolumn{8}{|l|}{ Maximum } & 1.72 & & 6.01 \\
\hline \multicolumn{8}{|l|}{ Minimum } & 0.01 & & 0.04 \\
\hline
\end{tabular}

Note. Base set used in Chiu (1995), experiments 1-24. 
Table 2. Comparison of the benchmark, exact and approximate $(Q, r)$ policies for $\lambda=10, L=1, \tau=3$, and $h=1$.

\begin{tabular}{|c|c|c|c|c|c|c|c|c|c|c|}
\hline Problem no. & $\pi$ & $p$ & $\hat{K}$ & $c$ & $\left(Q^{*}, r^{*}, T^{*}\right)_{\mathrm{bm}}$ & $T C_{\mathrm{bm}}^{*}$ & $\left(Q^{*}, r^{*}\right)_{e}$ & $\% \Delta_{e}$ & $\left(Q^{*}, r^{*}\right)_{a}$ & $\% \Delta_{a}$ \\
\hline 25 & 20 & 5 & 200 & 5 & $(29,7,0.96)$ & 149.13 & $(27,10)$ & 1.41 & $(37,0)$ & 15.78 \\
\hline 26 & 40 & 5 & 200 & 5 & $(29,11,1.08)$ & 154.92 & $(25,12)$ & 3.52 & $(28,10)$ & 7.06 \\
\hline 27 & 20 & 15 & 200 & 5 & $(26,9,1.08)$ & 153.43 & $(26,9)$ & 0.99 & $(35,0)$ & 18.02 \\
\hline 28 & 40 & 15 & 200 & 5 & $(26,11,1.08)$ & 161.15 & $(25,12)$ & 1.91 & $(26,10)$ & 4.10 \\
\hline 29 & 20 & 5 & 200 & 15 & $(26,0,0)$ & 235.33 & $(26,0)$ & 0.00 & $(33,0)$ & 5.69 \\
\hline 30 & 40 & 5 & 200 & 15 & $(26,10,1.08)$ & 258.50 & $(26,11)$ & 1.12 & $(28,8)$ & 3.79 \\
\hline 31 & 20 & 15 & 200 & 15 & $(25,0,0)$ & 236.85 & $(25,0)$ & 0.00 & $(32,0)$ & 7.05 \\
\hline 32 & 40 & 15 & 200 & 15 & $(26,10,1.08)$ & 262.05 & $(24,11)$ & 0.89 & $(27,8)$ & 3.13 \\
\hline \multicolumn{8}{|c|}{ Overall average } & 0.60 & & 3.23 \\
\hline \multicolumn{8}{|c|}{ Overall standard deviation } & 0.78 & & 4.28 \\
\hline \multicolumn{8}{|c|}{ Overall median } & 0.24 & & 1.63 \\
\hline Overall max & & & & & & & & 3.52 & & 18.02 \\
\hline \multicolumn{8}{|c|}{ Overall minimum } & 0.00 & & 0.04 \\
\hline
\end{tabular}

Notes. Large fixed ordering cost set, experiments 25-32. Reported statistics are for all 32 cases tested.

respect to the more complex time-based policy. Comparison of cost components shows that expected perishing costs are typically larger under the $(Q, r, T)_{\mathrm{bm}}$ policy, whereas expected lost-sales costs are typically smaller. It appears that the robustness of the $(Q, r)$ policy can be explained through some ratio of costs of expected shortage and perishing. To this end, we construct the following newsvendortype service-level measure,

$$
\begin{aligned}
\rho= & {\left[\int_{0}^{\tau}(\pi-c) \mathrm{E}[L S \mid Z=z] d F(z)\right] / } \\
& {\left[\int_{0}^{\tau}[(\pi-c) \mathrm{E}[L S \mid Z=z]+(p+c) \mathrm{E}[P \mid Z=z]] d F(z)\right] }
\end{aligned}
$$

evaluated at $Q^{*}$ and $r^{*}$.

In Figure 2, we plot $\% \Delta_{e}$ and $\% \Delta_{a}$ versus $\rho$ for each case considered. The exact determination of the $(Q, r)$ policy parameters yields a percentage error of less than $0.5 \%$

Figure 2. Performance of the exact and approximate computations of the $(Q, r)$ policy parameters vs. $\rho$.

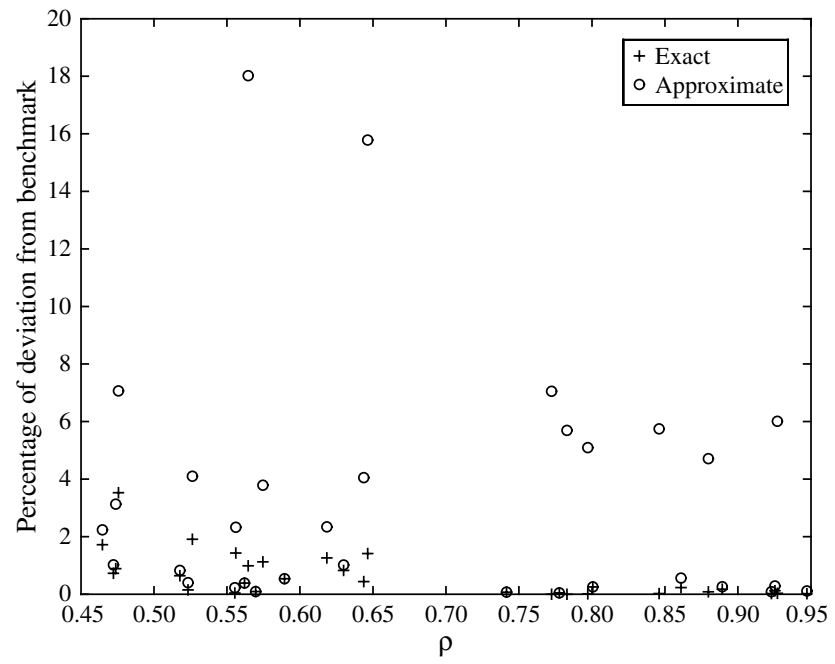

when $\rho$ is roughly larger than 0.75 , but for $\rho$ values less than 0.75 , the $(Q, r)$ policy starts performing badly vis-à-vis the $(Q, r, T)_{\mathrm{bm}}$ policy. This implies that the lot size-reorder point policy is not suitable for large $\rho$ values, which occur when the expected shortage costs are large compared to the expected holding costs under the best $(Q, r)$ policy. However, when fixed ordering costs are large, unit shortage or perishing costs are small, and shelf lives are relatively long, the $(Q, r)$ policy appears overall to be a good heuristic policy.

\section{Conclusion}

In this paper, we developed an analytical model for perishables with constant life times and replenishment lead times in the presence of nonnegligible ordering costs under the $(Q, r)$ control policy with $r<Q$. As a solution methodology, we introduced the concept of effective shelf life and the inventory system was characterized through a continuous valued state space embedded Markov chain. Establishing the ergodicity of the underlying effective shelf life process, we obtained its stationary distribution. The operating characteristics of the inventory system and the steady-state cost rate were then developed. Our numerical study indicates that the $(Q, r)$ policy is a reasonably good control policy when compared against a more complex, time-based policy except for high service levels. We also observed that computation of the $(Q, r)$ policy parameters exactly as modeled herein can result in significant cost savings. There are a number of possible extensions to our model, such as allowing for batch demands and multiple outstanding orders, which we leave as future work.

\section{Electronic Companion}

An electronic companion to this paper is available as part of the online version that can be found at http://or.journal. informs.org/. 


\section{Acknowledgments}

This research was partially funded by TUBITAK grant MISAG-104.

\section{References}

Chiu, H. N. 1995. An approximation to the continuous review inventory model with perishable items and lead times. Eur. J. Oper. Res. 87 93-108.

Fries, B. 1975. Optimal ordering policy for a perishable commodity with fixed lifetime. Oper. Res. 23 46-61.

Gürler, Ü., B. Y. Özkaya. 2003. A note on continuous review perishable inventory systems: Models and heuristics. IIE Trans. 35 321-323.

Laslett, G. M., D. B. Pollard, R. L. Tweedie. 1978. Techniques for establishing ergodic and recurrence properties of continuous-valued Markov chains. Naval Res. Logist. Quart. 25 455-472.
Lian, Z., L. Liu. 2001. Continuous review perishable inventory systems: Models and heuristics. IIE Trans. 33 809-822.

Liu, L., Z. Lian. 1999. $(s, S)$ continuous review model for products with fixed lifetimes. Oper. Res. 47 150-158.

Nahmias, S. 1975. Optimal ordering policies for perishable inventory. Oper. Res. 23 735-749.

Perry, D., M. J. M. Posner. 1998. An $(S-1, S)$ inventory system with fixed shelflife and constant lead time. Oper. Res. 46 S565-S571.

Ross, S. 1970. Average cost semi-Markov decision processes. J. Appl. Probab. 7 649-656.

Schmidt, C. P., S. Nahmias. 1985. $(S-1, S)$ policies for perishable inventory. Management Sci. 31 719-728.

Tijms, H. C. 1994. Stochastic Models: An Algorithmic Approach. Wiley, Chichester, UK.

Weiss, H. 1980. Optimal ordering policies for continuous review perishable inventory models. Oper. Res. 28 365-374. 